\title{
LOS TIPOS DE TRABAJO PRÁCTICO EXPERIMENTAL COMO HERRAMIENTAS PARA MEJORAR LAS PRÁCTICAS
}

\author{
Darwin Leonardo Vargas Sánchez ${ }^{1}$ leovargas18@gmail.com
}

Resumen

La química, como una ciencia experimental, tiene un componente práctico que debe ser trabajado utilizando prácticas de laboratorios o talleres que permitan a los estudiantes comprender los principios y teorías científicas analizando hechos cotidianos. Desde la enseñanza de las ciencias se proponen diferentes modelos de trabajo práctico experimental, cada uno de estos tienen como finalidad desarrollar habilidades en los estudiantes con el fin de mejorar su comprensión de los fenómenos a través de la química.

Palabras Clave

Química, prácticas de laboratorio, taller práctico, trabajo práctico, enseñanza de las ciencias.

\footnotetext{
${ }^{1}$ Estudiante del Departamento de Química de la UPN
} 
En los procesos de enseñanza y aprendizaje de la química, es muy importante contar con actividades experimentales como los laboratorios y los talleres prácticos, estas actividades les dan, a los estudiantes, herramientas que les permiten la comprensión de los principios y teorías científicas que se les quiere dar a conocer. En la educación secundaria, estos laboratorios y talleres prácticos se deben trabajar con metodologías llamativas, ya que la ciencia se les debe mostrar, contextualizada, aplicada y de fácil acceso. En el colegio Liceo Femenino se observa que las estudiantes reciben una buena fundamentación teórica de los diferentes temas de la química, pero las prácticas de laboratorio son tradicionales, no contextualizadas, esto hace que las estudiantes no comprendan completamente los conceptos y se desmotiven con la asignatura, por lo anterior se propone trabajar el siguiente problema: ¿EI diseño y aplicación de prácticas de laboratorio y de talleres útiles, mejorarán el interés, la motivación y el aprendizaje de la química en estudiantes de grado once?

\section{Objetivos}

Para el desarrollo de esta propuesta de investigación, se proponen los siguientes objetivos:

\section{General}

$\diamond \quad$ Diseñar y desarrollar prácticas de laboratorio y talleres útiles para facilitar, a las estudiantes, la comprensión de las teorías y de los conceptos químicos.

\section{Específicos}

$\checkmark \quad$ Caracterizar las prácticas de laboratorio que se realizan en el colegio Liceo Femenino.

$\diamond \quad$ Desarrollar, en las estudiantes, habilidades prácticas y procesos procedimentales en el trabajo práctico realizado en química.

$\checkmark \quad$ Desarrollar, en las estudiantes, habilidades en la investigación para comprender como funciona las ciencia

\section{Referente teórico}

Los trabajos prácticos, sin duda alguna, son de las actividades más importantes en la enseñanza de las ciencias, generalmente los colegios e instituciones de educación media desarrollan trabajos prácticos ineficientes, puesto que se presentan a los estudiantes con un formato cerrado, es decir como un conjunto de instrucciones que los estudiantes deben de seguir sin darles la oportunidad para que se den cuenta del problema que hay que resolver.

Teniendo en cuenta todos los aspectos anteriormente mencionados surge una propuesta didáctica que ha sido trabajada por algunos pedagogos y didactas, como Woolnough y Alsop (1985), Gott I Duggan (1995), Caamaño (2007) y Molina, Carriazo y Farías (2009), esta propuesta didáctica pretende que los 
trabajos prácticos de laboratorio, que se realizan en las escuelas y colegios, aborden problemas significativos en el desarrollo de modelos teóricos escolares. Molina, Carriazo Y Farías (2009), definen los trabajos prácticos de laboratorio como toda actividad en la que el alumno está implicado y no necesita estar necesariamente en un laboratorio o con material o equipo especial para aprender, Caamaño (2007) propone clasificar los trabajos prácticos de la siguiente manera:

-Experiencias: son imágenes reales o representativas que sirven para obtener un acercamiento de tipo perceptivo con los fenómenos, con estos se puede: adquirir una experiencia directa con los sentidos sobre los fenómenos científicos, lo que permite plantear una relación entre teoría y realidad, adquirir conocimientos de forma potencial para ser útiles en la resolución de problemas. Las actividades prácticas, desarrolladas bajo este tipo de trabajo, tienen finalidades exploratorias sobre las ideas de los estudiantes.

-Experimentos ilustrativos: Se utilizan para enlazar una evidencia experimental con el aprendizaje de conceptos o para ilustrar leyes o principios, permiten interpretar un fenómeno, ilustrar un principio o mostrar una relación entre variables; con su aplicación se despierta la curiosidad de los estudiantes, antes y después, de la elaboración del experimento. Este tipo de trabajo práctico abre grandes posibili- dades para que se desarrollen discusiones dirigidas por el docente, en donde los estudiantes opinan sobre la interpretación de los fenómenos observados en clases.

-Ejercicios prácticos: Sirven para aprender a seguir protocolos tendientes a enseñar una destreza experimental, el uso de un equipo, un procedimiento especial de análisis o simplemente para seguir indicaciones, Los ejercicios prácticos pueden ser de dos tipos procedimentales y corroborativos, los procedimentales presuponen el aprendizaje por etapas, los corroborativos pretenden verificar una teoría o una ley por medio de un procedimiento detallado.

-Investigaciones: Es una actividad que busca acercar al estudiante a la forma como se produce el conocimiento científico, puede enfocarse a resolver problemas teóricos o prácticos, trata de acercar al estudiante al proceso de construcción de la ciencia, es decir, seguir el paso que utilizan los científicos para construir el conocimiento. En este tipo de investigación el estudiante actúa como un investigador novato y el profesor como el director de la investigación. Se pueden realizar investigaciones escolares de muchos tipos, como para resolver problemas prácticos procedimentales o para resolver problemas teóricos o simplemente para resolver problemas prácticos.

\section{Metodología}

El proyecto se realizará en cuatro etapas o fases

Diagnóstico

Diseño de actividades

Aplicación de los trabajos prácticos

Evaluación de la propuesta de intervención: 


\section{Diagnóstico}

Esta fase se desarrolla en dos momentos: en el primero se caracterizan las prácticas de laboratorio utilizadas en el colegio utilizando matriz de valoración de acuerdo con la propuesta de clasificación de tipos de trabajo práctico propuesto por Caamaño (2007); para clasificar los trabajos de laboratorio, se toman datos de las dos primeras prácticas de laboratorio realizadas en el primer periodo con los seis cursos de grado once. En estas prácticas se toma un registro detallado en un diario de campo.

En el segundo momento, se caracterizan las actitudes hacia la ciencia y razonamiento cognitivo de las estudiantes del grado once, se utilizan dos instrumentos, validados, con sus respectivas categorías de análisis y se unifican en uno solo para su aplicación.

- El primero de ellos es el Protocolo de las Actitudes hacia la Ciencia (PAC), es una adaptación hecha al español por Vásquez y Manassero (1995), desarrollada por Wareing en 1982. Este instrumento consta de 48 ítems en escala tipo Likert, agrupados en cuatro categorías: enseñanza de la ciencia, imagen de ciencia, social y conocimiento científico y técnico (Anexo 2).

- El segundo fue tomado de Pozo $Y$ Gómez (2001), (Anexo 3) que evalúa la habilidad cognitiva; se tomaron únicamente dos ítems, de los cuatro que contiene, los que se adaptaron para facilitar la resolu ción por parte de las estudiantes. Se considera que estos ítems son significativos para utilizarlos en el instrumento, ya que se ajustan a algunas temáticas desarrolladas.

\section{Diseño y aplicación de actividades}

Las prácticas de laboratorio están centradas en el desarrollo de experiencias en donde las estudiantes utilizan únicamente la observación. Se tabulan los resultados obtenidos de 58 estudiantes, quienes tienen facilidad para aprender los conceptos de las ciencias, sin embargo, no logran establecer las relaciones ciencia-tecnología-sociedad-ambiente. Las estudiantes, en cuanto a las habilidades cognitivas, se pueden ubicar en el realismo ingenuo.

Se diseña el plan de los temas que se trabajaran durante todo el año, en orden cronológico; teniendo este plan, se diseñan las prácticas de laboratorio y talleres prácticos, teniendo en cuenta los diferentes modelos de trabajo práctico experimental. Se desarrolla durante el tercer periodo del calendario escolar, se diseñan cinco talleres prácticos experimentales, se desarrollan los tres primeros, 2 correspondientes a experiencias y el último relacionado con experimentos ilustrativos. 


\begin{tabular}{|c|c|}
\hline Modalidad & Experiencia \\
\hline Nombre & Combustión \\
\hline Objetivos & $\begin{array}{l}\text { - } \quad \text { - Conocer combustibles en los tres estados de la materia. } \\
\text {-Comprender los procesos que se llevan a cabo en una reacción de } \\
\text { combustión. }\end{array}$ \\
\hline Momento de aplicación & $\begin{array}{l}\text { Este taller se aplicó cuando se estaba desarrollando el tema de reacciones de } \\
\qquad \begin{array}{c}\text { los hidrocarburos; se aplicó para facilitar la comprensión } \\
\text { de las reacciones de oxidación. }\end{array}\end{array}$ \\
\hline
\end{tabular}

\begin{tabular}{|c|c|}
\hline Modalidad & Experiencia \\
\hline Nombre & Polimerización \\
\hline Objetivos & $\begin{array}{l}\text { - Conocer algunas propiedades de los polímeros. } \\
\text { - Comprender los mecanismos y características de una reacción } \\
\text { de polimerización }\end{array}$ \\
\hline Momento de aplicación & $\begin{array}{l}\text { El taller se aplicó cuando se estaba desarrollando el tema de reacciones y } \\
\text { propiedades de los alquenos. }\end{array}$ \\
\hline
\end{tabular}

\begin{tabular}{|c|c|}
\hline \multicolumn{2}{|r|}{ Taller 3} \\
\hline Modalidad & Experimentos ilustrativos \\
\hline Nombre & Taller de origami molecular \\
\hline Objetivos & $\begin{array}{l}\text { Comprender la tetra valencia del átomo de carbono y las geome- } \\
\text { trías que puede presentar en los diferentes grupos funcionales } \\
\text { Nombrar correctamente los grupos funcionales }\end{array}$ \\
\hline Momento de aplicación & $\begin{array}{l}\text { Se aplicó cuando se trató el tema de las nomenclaturas } \\
\text { de los hidrocarburos. }\end{array}$ \\
\hline
\end{tabular}




\begin{tabular}{|c|c|}
\hline \multicolumn{2}{|c|}{ Taller $\mathbf{4}$} \\
\hline Modalidad & Ejercicios prácticos \\
\hline Nombre: & Extracción de aceites esenciales \\
\hline Objetivos & Comprender el concepto de solubilidad de \\
\hline los compuestos orgánicos
\end{tabular}

\section{RESULTADOS Y ANALISIS}

Se inicia el análisis, por categorías, de los resultados obtenidos en "Actitudes hacia la ciencia", que muestra cambios significativos en las actitudes de las estudiantes:

\section{Enseñanza de la ciencia}

En esta categoría, en el ítem 4 dice: la ciencia es muy difícil de aprender, la respuesta "de acuerdo" paso del $47,4 \%$ a $26.67 \%$, lo que indica que la propuesta permitió que las estudiantes vieran que las ciencias se pueden aprender fácilmente.

\section{imagen de ciencia}

En esta categoría las estudiantes empezaron a ver que la ciencia es muy útil y de aplicación, para comprender algunos fenómenos, esto se evidenció en el ítem 50 que dice: estudiar ciencia es muy útil, incluso cuando se terminan los estudios, allí la categoría "totalmente de acuerdo tuvo una variación de $34.4 \%$ a $56,7 \%$ ".

\section{Social}

En esta categoría las estudiantes empezaron a ver la ciencia más contextualizada y aplicada, Por ejemplo, en el ítem 20 que dice: conocer la luna y los planetas nos ayuda aquí en la tierra, se observó uno de los cambios más significativos puesto que la respuesta "de acuerdo" paso del $32.8 \%$ al $60 \%$

\section{Conocimiento científico y técnico}

Desde el punto de vista de esta categoría, las estudiantes empezaron a ver la ciencia como una herramienta que ayuda a mejorar las habilidades del pensamiento, esto se identifica en el ítem 27 que dice: la ciencia ayuda a pensar mejor, en donde la respuesta "totalmente de acuerdo pasó de $20.7 \%$ al $36,67 \% "$ 
El análisis de los resultados obtenidos en las habilidades cognitivas hacia las ciencias permite establecer que las estudiantes mejoraron considerablemente con respecto a la fase de diagnóstico, como se observa en las gráficas, en las que en la mayoría de preguntas las estudiantes dan respuestas que tienden al constructivismo, es decir, son respuestas sustentadas en modelos teóricos propuestos desde la química.

Con respecto a la pregunta número uno, que cuestionaba a las estudiantes sobre, "qué le pasa a la estructura química de los polímeros de un pañal cuando absorbe orina," la mayoría de las estudiantes explicó este fenómeno por la absorción de la orina por el polímero sin modificar su estructura, haciendo que aumente su peso y su volumen. Muchas realizaban la comparación de este fenómeno con las bolitas de hidrogel que se utilizan para las plantas, explicaban que las bolitas tienen un tamaño cuando están deshidratadas, pero cuando absorben agua del medio circundante aumentan de volumen sin modificar su estructura. A continuación se muestra la respuesta que propuso una estudiante:" El pañal cuenta con unas partículas llamadas polímeros que absorben la orina aumentando su tamaño como las pelotas de hidrogel"

\section{PREGUNTA 1}

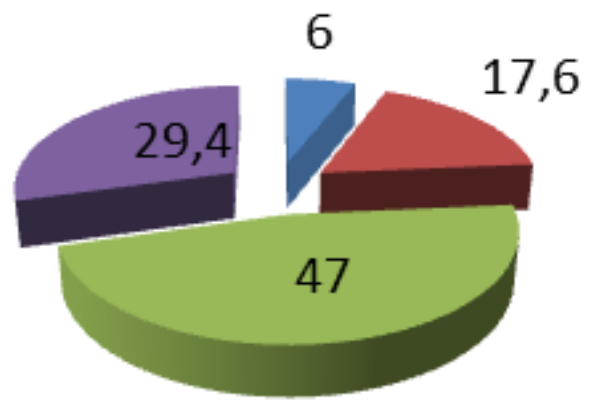

Realismo
Ingenuo
Realismo
Interpretativo
Constructivimo 
Con respecto a la pregunta número dos que cuestionaba a las estudiantes, "qué sucede cuando una varilla de vidrio es frotada con una bayetilla y luego se acerca a un chorro de agua de caída constante y se observa que este es atraído," en general la mayoría de estudiantes explicaron este fenómeno desde las cargas eléctricas, afirmando que la varilla de vidrio queda cargada positivamente y el agua como tiene electrones libres es atraído por el polo opuesto, de esta manera se explica cómo se desvía el recorrido del agua, A continuación se muestra la respuesta que propuso una estudiante: "La varita al ser cargada positivamente y acercarla al agua, que esta cargada negativamente, hay una fuerza de atracción entre los dos polos opuestos"

\section{PREGUNTA 2}

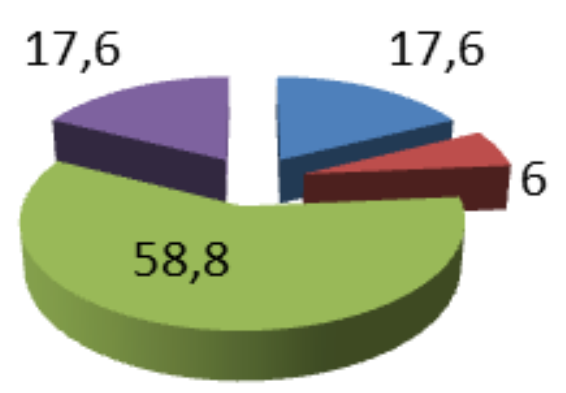

- Realismo Ingenuo

- Realismo Interpretativo

Constructivimo 
Con respecto a la pregunta número tres, que cuestionaba a las estudiantes "por qué la sal de cocina y el azúcar presentan diferentes geometrías moleculares," la gran mayoría de las estudiantes explica, que por la composición y los enlaces que tiene cada una de las moléculas, tienen una geometría diferente, en el caso del azúcar, algunas estudiantes hablan de la geometría molecular dependiendo del número de átomos de carbono que presente la molécula. A continuación se muestra la respuesta que propuso una estudiante "Esto se debe a los elementos que componen cada sustancia, en el caso del azúcar $\mathrm{C}, \mathrm{O}$ y $\mathrm{H}$ y la sal $\mathrm{Na}$ y $\mathrm{Cl}$, depende del tipo de enlace y así mismo del número de átomos de carbono"

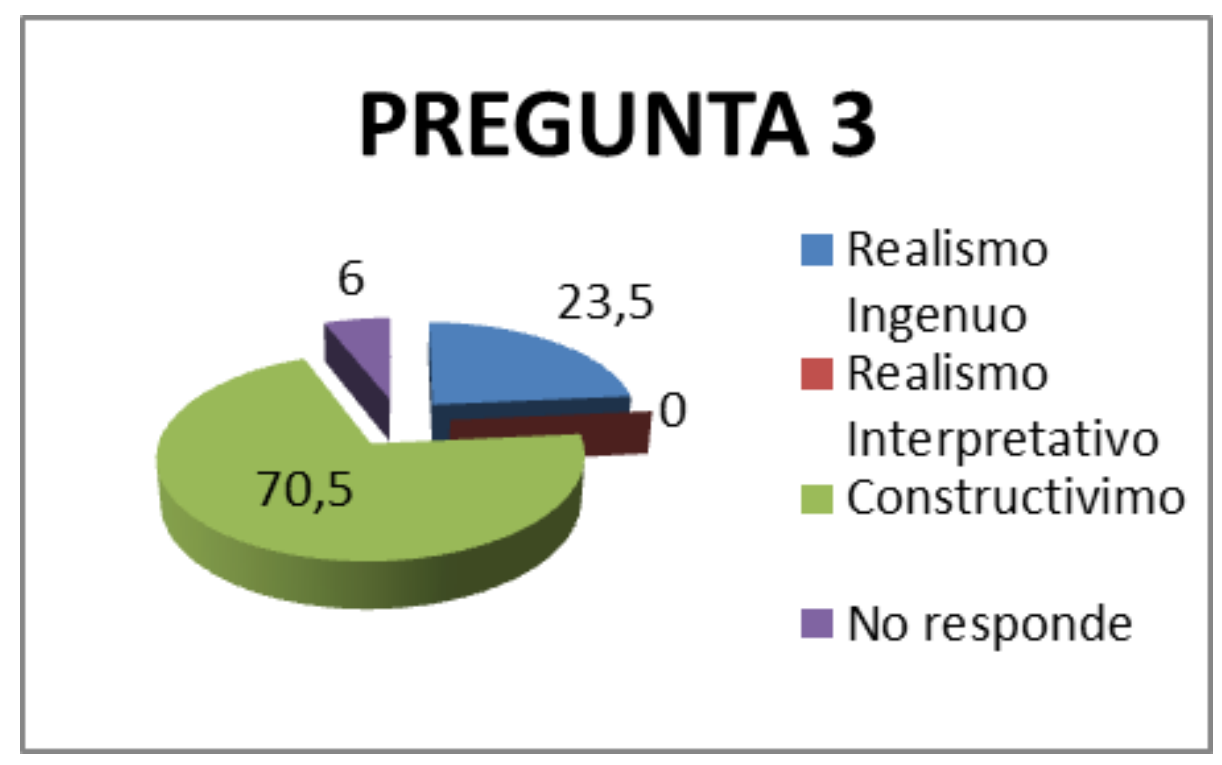




\section{Conclusiones}

\section{Con respecto a los tipos}

de trabajo práctico:

De los tipos de trabajo práctico se puede concluir:

$\checkmark \quad$ Son una buena estrategia para organizar el trabajo de laboratorio, puesto que las experiencias, los experimentos ilustrativos, los ejercicios prácticos y las investigaciones desarrollan diferentes habilidades en los estudiantes.

$\checkmark \quad$ Se consolidan como un modelo muy eficaz para reemplazar a las prácticas de laboratorio cerradas y monótonas, en donde los estudiantes no comprenden el problema y por lo tanto no pueden hacer parte de la solución.

Son una buena herramienta para complejizar el conocimiento de las estudiantes, puesto que su aplicación permite identificar concepciones alternativas, dificultades de aprendizaje y al mismo tiempo, emprender acciones con las estudiantes para que se superen las dificultades y se reconcilien las concepciones alternativas con el conocimiento científico .

Con respecto a las estudiantes y al profesorado

Los talleres utilizados permitieron identificar algunas concepciones alternativas y errores conceptuales que las estudiantes presentaban en algunas temáticas de la química, los talleres aplicados fueron de gran ayuda para superar estas dificultades. mental y los talleres diseñados bajo esta estrategia, tienen alta incidencia en el aprendizaje de las estudiantes..

$\checkmark \quad$ Los tipos de trabajo práctico experimental permiten al docente organizar el trabajo de laboratorio de una manera más eficaz.

\section{Bibliografía}

-Caamaño. A. (2005) Trabajos prácticos investigativos en química en relación con el modelo atómico-molecular de la materia, planificados mediante un dialogo estructurado entre profesor y estudiantes. Revista Educación química 16 [1],

- Jiménez, Caamaño, Oñorbe y Pedrinacci (2007).Enseñar ciencias, Serie didáctica de las ciencias experimentales, editorial Grao, Segunda edición.

-Molina, Carriazo y Farias.(2009)Taller sobre el uso de los tipos de trabajo practico como herramienta fundamental para enseñar ciencias. Tecne Episteme $\mathrm{Y}$ Didaxis. $\mathrm{N}^{\mathrm{a}}$ Extraordinario.

-Pozo y Gómez.(2001) Aprender y enseñar ciencia, Del conocimiento cotidiano al conocimiento científico, Morata ediciones, Tercera edición.

-Rodríguez, Jiménez y Caicedo (2007).Protocolo de actitudes relacionadas con la ciencia: Adaptación para Colombia, Phychologia, Avances en la disciplina, Vol. 1, Na2:85100. 


\section{Anexo 2}

\section{Test a estudiantes}

Este instrumento está diseñado para valorar sus actitudes hacia la ciencia. No existen respuestas correctas o incorrectas, sólo se desea conocer su opinión sincera sobre cada afirmación. Por favor, lea atentamente cada frase y señale con una (X) así:

TA = Totalmente de acuerdo. $A=$ De acuerdo. NS = No estoy seguro. $\mathbf{D}=$ En desacuerdo. TD = Totalmente en desacuerdo.

1.Gracias a la ciencia tenemos un mundo mejor

2.La ciencia no le gusta a nadie

3.La ciencia nos ayuda a ahorrar tiempo y esfuerzo

4.La ciencia es muy difícil de aprender

5.Gracias a la ciencia las enfermedades se pueden curar

6. Entre más conocimiento científico existe más preocupaciones hay para nuestro mundo.

7.La ciencia no es aburrida

8.La ciencia ayuda a la gente en todos los lugares

9. La ciencia es lógica

10.No me gusta pensar en la ciencia

11.La curiosidad es lo primordial se la ciencia

12.Gracias a la ciencia la gente tiene más salud

13. La ciencia nos soluciona los problemas energéticos

14. Para destacarse en ciencia es necesario ser muy inteligente

15.Los alumnos estudian ciencia porque es obligatorio

16. La ciencia es el medio para conocer el mundo donde vivimos

17. La ciencia estimula la curiosidad

18. Trabajar en ciencias es mejor que trabajar en otras áreas.

19. La ciencia es muy valiosa

20. Conocer científicamente la luna y los planetas nos ayuda aquí en la tierra.

21. Las clases de ciencia son monótonas

22.Las asignaturas de ciencias son las peores

23. No deberían existir asignaturas de ciencias

24.La gente vive más gracias a la ciencia

25.En las clases de ciencia los alumnos hacen las cosas mecánicamente

26. La ciencia disminuye la curiosidad

27. La ciencia ayuda a pensar mejor

28. Estudiar ciencias es aburrido

29.Los alumnos serian mejores estudiantes sino tuvieras que estudiar ciencias

30. La ciencia solo tiene sentido para los científicos 


\section{Continuación}

\section{Anexo 2}

\section{Test a estudiantes}

\begin{tabular}{|c|c|c|c|c|c|}
\hline Afirmación & TA & A & NS & D & TD \\
\hline \multicolumn{6}{|l|}{ 31.La ciencia ayuda a prevenir catástrofes } \\
\hline \multicolumn{6}{|l|}{ 32. Con la ciencia tendremos un mundo mejor } \\
\hline \multicolumn{6}{|l|}{ 33.La ciencia nos enseña a prepararnos para el futuro } \\
\hline \multicolumn{6}{|l|}{ 34.La ciencia pone en riesgo la salud } \\
\hline \multicolumn{6}{|l|}{ 35.La vida sería aburrida sin los aportes de la ciencia } \\
\hline \multicolumn{6}{|l|}{ 36.No se debió haber enviado gente a la luna } \\
\hline \multicolumn{6}{|l|}{ 37. La ciencia es muy aburrida } \\
\hline \multicolumn{6}{|l|}{ 38.La ciencia es un pretexto para manipular } \\
\hline \multicolumn{6}{|l|}{ 39.La ciencia es desagradable } \\
\hline \multicolumn{6}{|l|}{ 40.La ciencia es muy útil } \\
\hline \multicolumn{6}{|l|}{ 41.La ciencia es muy necesaria } \\
\hline \multicolumn{6}{|l|}{ 42.Estudiar ciencia satisface la curiosidad } \\
\hline \multicolumn{6}{|l|}{ 43.La ciencia no es útil } \\
\hline \multicolumn{6}{|l|}{ 44.La ciencia no ayuda a aceptar opiniones diferentes } \\
\hline \multicolumn{6}{|l|}{ 45.En la ciencia es importante tener en cuenta las ideas nuevas } \\
\hline \multicolumn{6}{|l|}{ 46.El conocimiento científico no se puede modificar } \\
\hline \multicolumn{6}{|l|}{ 47.La ciencia es muy interesante } \\
\hline 48.Estudiar ciencia es muy útil incluso cuando se terminan los estudios & & & & & \\
\hline
\end{tabular}

\section{Categorías de análisis}

\begin{tabular}{|l|c|c|}
\hline \multirow{2}{*}{ Categoría de enseñanza } & Categoría Enseñanza, & $\begin{array}{c}\text { Resultado de la enseñanza de la ciencia } \\
\text { ítems: } 4,14,15,21,22,23,25,\end{array}$ \\
\cline { 2 - 2 } & & Ciencia Escolar, ítems: 4, 15, 21, 22, 23 y 28. \\
\hline
\end{tabular}

Categoría de Social

\begin{tabular}{|l|c|l|}
\hline \multirow{3}{*}{ Categoría característica } & Ítems:9, 11, 16, 17, 26, 27, & Naturaleza, ítems: 9, 16, 27, 45, 47 y 48. \\
\cline { 3 - 3 } & $42,44,45,46,47$ y 48. & Curiosidad, ítems: $11,17,26$ y 42 \\
\cline { 3 - 3 } & & Colectiva, ítems 44 y 46. \\
\hline
\end{tabular}




\section{Anexo 3 \\ Instrumento de evaluación \\ Categoría Cognitiva}

Responda a cada una de las siguientes preguntas, si es necesario, se puede ayudar de gráficos o dibujos para su explicación.

1. Un pañal tiene la propiedad de absorber la orina gracias a algunas partículas gelatinosas o polímeros que lo conforman, estas partículas pueden crecer hasta 60 veces su tamaño original dependiendo de la cantidad de orina absorbida. ¿Cómo se puede explicar este proceso de absorción en el pañal teniendo en cuenta la estructura química del polímero? Realiza un dibujo para explicar su respuesta.

2. Una varilla de vidrio es cargada eléctricamente por fricción con una bayetilla, luego es acercada a una llave de agua con caída constante y se observa que el agua desvía su recorrido debido a una atracción hacia la varilla de vidrio ¿Cómo puede explicar este fenómeno? Realice un dibujo si lo considera necesario.

3. Luego de cristalizar algunas sustancias como la sal del cocina y el azúcar se observan cristales con formas cúbicas y hexagonales ¿Por qué estas sustancias presentan geometrías diferentes? ¿Cómo se pueden explicar estas diferencias?

Categorías de análisis.

\begin{tabular}{|c|c|}
\hline \multirow{3}{*}{ Categoría cognitiva } & $\begin{array}{l}\text { Realismo ingenuo: La estudiante da explicaciones ingenuas sin utilizar términos, modelos o } \\
\text { teorías científicas, responde de acuerdo con sus creencias, que generalmente son ingenuas. }\end{array}$ \\
\hline & $\begin{array}{l}\text { Realismo interpretativo: La estudiante explica los fenómenos a partir de lo que puede } \\
\text { percibir con los sentidos, pero no logra explicar molecular o electrónicamente } \\
\text { los fenómenos que evidencia. }\end{array}$ \\
\hline & $\begin{array}{l}\text { Constructivismo: La estudiante explica los fenómenos desde deferentes modelos de la } \\
\text { química con los que se interpreta la realidad. }\end{array}$ \\
\hline
\end{tabular}

